\title{
Review of: "Dominance of the residential sector in Chinese black carbon emissions as identified from downwind atmospheric observations during the COVID-19 pandemic"
}

\author{
Yasunori Tohjima
}

Potential competing interests: The author(s) declared that no potential competing interests exist.

In this study, Kanaya and colleagues examined the BC and CO observed at Fukue atmospheric environment observatory during Februar-March to partition their sources into residential and nonresidential sources. During the winter-spring period, the observed BC and CO concentrations often show the elevated values when the air masses are transported from the northern and eastern China. Comparing such elevated values with the model simulated values, the authors evaluated the temporal changes in the $\mathrm{BC}$ and $\mathrm{CO}$ fluxes in China. Additionally, it was estimated that the substantial reductions of the BC and CO emissions from the industry and transport sectors during February to March 2020 due to the COVID-19 lockdown in China. Taking the opportunity, the authors evaluated the contribution of the non-residential sector by attributing the decrease in the fluxes in 2020 to the non-residential sector.

The idea and method adopted in this study is appropriate and the result is very convincing. I think that this study would contribute to the mitigation policy for the reduction of $\mathrm{BC}$ emissions in China.

Although I found that this paper is excellent, I have following two comments:

1. In this study, the authors assumed that the fluxes in 2020 would have been almost same as those in 2019 if the COVID-19 outbreak had not occurred. This assumption seems to be valid for the CO flux because of the rather stable CO fluxes during 2015-2019 period as shown in Fig. 3e. However, the BC fluxes showed considerable variations: abrupt increase in 2016 and gradual decrease during 2016-2019. I think it would be better to discuss the cause of these variations in the manuscript. The authors attributed the gradual decrease in the BC flux during 2016-2019 to the non-residential sector. If so, the nonresidential contribution of the BC flux reached to about 50\% in 2016 and 2017. This sectoral contribution is consistent with the bottom-up estimation of MEIC, CEDS, and REAS listed in Table 1. I think it would be better to describe these results in the manuscript.

2. The authors limited the period for the analysis to February and March. However, I think it would be better to examine the flux variations between before and after COVID-19 lockdown in China, that is for example between December 2019 and May 2020. It was estimated that the socioeconomic activity in 
China was not so confined before February 2020 and was close to the normal level after March 2020.

Therefore, I believe that the temporal variations in the fluxes for the above period is very intriguing. This approach could simply evaluate the COVID-19 impact on the BC fluxes because the influences of the longterm BC flux decreasing trend is limited. 